\title{
Existence results for m-point boundary value problems of nonlinear fractional differential equations with $p$-Laplacian operator
}

\section{Zhi-Wei LV}

"Correspondence:

sdlllzw@mail.ustc.edu.cn Department of Mathematics,

Zhengzhou University, Zhengzhou, Henan 450001, P.R. China

Department of Mathematics and

Physics, Anyang Institute of

Technology, Anyang, Henan

455000, P.R. China

\begin{abstract}
In this paper, we discuss the existence and multiplicity of positive solutions to $m$-point boundary value problems of nonlinear fractional differential equations with p-Laplacian operator$$
\left\{\begin{array}{l}
D_{0+}^{\beta}\left(\varphi_{p}\left(D_{0+}^{\alpha} u(t)\right)\right)+\varphi_{p}(\lambda) f(t, u(t))=0, \quad 0<t<1, \\
u(0)=0, \quad D_{0+}^{\gamma} u(1)=\sum_{i=1}^{m-2} \xi_{i} D_{0+}^{\gamma} u\left(\eta_{i}\right), \quad D_{0+}^{\alpha} u(0)=0,
\end{array}\right.
$$

where $D_{0+,}^{\alpha}, D_{0+}^{\beta}$ and $D_{0+}^{\gamma}$ are the standard Riemann-Liouville fractional derivatives with $1<\alpha \leq 2,0<\beta, \gamma \leq 1,0 \leq \alpha-\beta-1, \lambda \in(0,+\infty), 0<\xi_{i}, \eta_{i}<1, i=1,2, \ldots, m-2$, $\sum_{i=1}^{m-2} \xi_{i} \eta_{i}^{\alpha-\beta-1}<1,0 \leq \alpha-\gamma-1, f \in C([0,1] \times[0,+\infty),[0,+\infty))$, and $\varphi_{p}(s)=|s|^{p-2} s$, $p>1, \varphi_{p}^{-1}=\varphi_{q}, \frac{1}{p}+\frac{1}{q}=1$. Our results are based on the monotone iterative technique and the theory of the fixed point index in a cone. Furthermore, two examples are also given to illustrate the results.
\end{abstract}

Keywords: fractional differential equation; m-point boundary value problems; $p$-Laplacian operator

\section{Introduction}

Fractional differential equations arise in various areas of science and engineering. Due to their applications, fractional differential equations have gained considerable attention (see, e.g., [1-26] and the references therein).

Recently, there have been some papers dealing with the existence of solutions for nonlinear fractional differential equations with $p$-Laplacian operator. In [1], Wang et al. investigated the following boundary value problem for fractional differential equations with $p$-Laplacian operator:

$$
\left\{\begin{array}{l}
D_{0+}^{\beta}\left(\varphi_{p}\left(D_{0+}^{\alpha} u(t)\right)\right)+f(t, u(t))=0, \quad 0<t<1, \\
u(0)=0, \quad u(1)=a u(\xi), \quad D_{0+}^{\alpha} u(0)=0, \quad D_{0+}^{\alpha} u(1)=b D_{0+}^{\alpha} u(\eta),
\end{array}\right.
$$

where $D_{0+}^{\alpha}, D_{0+}^{\beta}$ are the standard Riemann-Liouville fractional derivatives, $1<\alpha, \beta \leq 2$, $0 \leq a, b \leq 1,0<\xi, \eta<1, f(t, u) \in C[(0,1) \times(0,+\infty),[0,+\infty)]$.

O2014 Lv; licensee Springer. This is an Open Access article distributed under the terms of the Creative Commons Attribution License (http://creativecommons.org/licenses/by/2.0), which permits unrestricted use, distribution, and reproduction in any medium, provided the original work is properly cited. 
In [2], Chai studied the existence of positive solutions of the following fractional differential equations with $p$-Laplacian operator:

$$
\left\{\begin{array}{l}
D_{0+}^{\beta}\left(\varphi_{p}\left(D_{0+}^{\alpha} u(t)\right)\right)+f(t, u(t))=0, \quad 0<t<1, \\
u(0)=0, \quad u(1)+\sigma D_{0+}^{\gamma} u(1)=0, \quad D_{0+}^{\alpha} u(0)=0,
\end{array}\right.
$$

where $D_{0_{+}}^{\alpha}, D_{0+}^{\beta}$ and $D_{0_{+}}^{\gamma}$ are the standard Riemann-Liouville fractional derivatives with $1<\alpha \leq 2,0<\beta \leq 1,0<\gamma \leq 1,0 \leq \alpha-\gamma-1$, the constant $\sigma$ is a positive number, $f(t, u) \in$ $C\left(I \times \mathbb{R}_{+}, \mathbb{R}_{+}\right)$.

In [3], Chen and Liu studied the following fractional differential equations with $p$ Laplacian operator:

$$
\left\{\begin{array}{l}
D_{0+}^{\beta}\left(\varphi_{p}\left(D_{0+}^{\alpha} x(t)\right)\right)=f(t, x(t)), \quad t \in[0,1] \\
x(0)=-x(1), \quad D_{0+}^{\alpha} x(0)=-D_{0+}^{\alpha} x(1)
\end{array}\right.
$$

where $0<\alpha, \beta \leq 1,1<\alpha+\beta \leq 2, D_{0+}^{\alpha}, D_{0+}^{\beta}$ are Caputo fractional derivatives, and $f:[0,1] \times$ $\mathbb{R} \rightarrow \mathbb{R}$ is continuous.

In [4], Lu et al. studied the following fractional differential equations with $p$-Laplacian operator:

$$
\left\{\begin{array}{l}
D_{0+}^{\beta}\left(\varphi_{p}\left(D_{0+}^{\alpha} u(t)\right)\right)=f(t, u(t)), \quad t \in[0,1] \\
u(0)=u^{\prime}(0)=u^{\prime}(1)=0, \quad D_{0+}^{\alpha} u(0)=D_{0+}^{\alpha} u(1)=0
\end{array}\right.
$$

where $2<\alpha \leq 3,1<\beta \leq 2, D_{0_{+}}^{\alpha}, D_{0_{+}}^{\beta}$ are the standard Riemann-Liouville fractional derivatives, and $f(t, u) \in C([0,1] \times[0,+\infty),[0,+\infty))$.

On the other hand, in [5], Bai studied an eigenvalue interval of the following fractional boundary problem:

$$
\left\{\begin{array}{l}
{ }^{c} D_{0+}^{\alpha} u(t)+\lambda h(t) f(u(t))=0, \quad 0<t<1, \\
u(0)=u^{\prime}(1)=u^{\prime \prime}(0)=0,
\end{array}\right.
$$

where $2<\alpha \leq 3,{ }^{c} D_{0+}^{\alpha}$ is the standard Caputo fractional derivative, $\lambda>0$.

In [6], Zhang et al. studied the following singular eigenvalue problem for a higher order fractional differential equation:

$$
\left\{\begin{array}{l}
-D^{\alpha} x(t)=\lambda f\left(x(t), D^{\mu_{1}} x(t), D^{\mu_{2}} x(t), \ldots, D^{\mu_{n-1}} x(t)\right), \quad 0<t<1, \\
x(0)=0, \quad D^{\mu_{i}} x(0)=0, \quad D^{\mu} x(1)=\sum_{j=1}^{p-2} a_{j} D^{\mu} x\left(\xi_{j}\right), \quad 1 \leq i \leq n-1,
\end{array}\right.
$$

where $n \geq 3, n-1<\alpha \leq n, n-l-1<\alpha-\mu_{l}<n-1$ for $l=1,2, \ldots, n-2$, and $\mu-\mu_{n-1}>0$, $\alpha-\mu_{n-1} \leq 2, \alpha-\mu>1$. $D_{0+}^{\alpha}$ is the standard Riemann-Liouville fractional derivative.

Moreover, in recent years, we have done some work on fractional differential equations [7-9]. In [7], we considered the following m-point boundary value problem for fractional differential equations:

$$
\left\{\begin{array}{l}
D_{0+}^{\alpha} u(t)+f(t, u(t))=0, \quad 0<t<1 \\
u(0)=0, \quad D_{0+}^{\beta} u(1)=\sum_{i=1}^{m-2} \xi_{i} D_{0+}^{\beta} u\left(\eta_{i}\right)
\end{array}\right.
$$


where $D_{0+}^{\alpha}$ is the standard Riemann-Liouville fractional derivative, $n=[\alpha]+1, f:[0,1] \times$ $[0, \infty) \rightarrow[0, \infty)$ is continuous, $1<\alpha \leq 2,0 \leq \beta \leq 1,0 \leq \alpha-\beta-1,0<\xi_{i}, \eta_{i}<1, i=$ $1,2, \ldots, m-2$, and $\sum_{i=1}^{m-2} \xi_{i} \eta_{i}^{\alpha-\beta-1}<1$.

Combining our work, in this paper, we discuss the existence of positive solutions for the following fractional differential equations with $p$-Laplacian operator:

$$
\left\{\begin{array}{l}
D_{0+}^{\beta}\left(\varphi_{p}\left(D_{0+}^{\alpha} u(t)\right)\right)+\varphi_{p}(\lambda) f(t, u(t))=0, \quad 0<t<1, \\
u(0)=0, \quad D_{0+}^{\gamma} u(1)=\sum_{i=1}^{m-2} \xi_{i} D_{0+}^{\gamma} u\left(\eta_{i}\right), \quad D_{0+}^{\alpha} u(0)=0,
\end{array}\right.
$$

where $D_{0+}^{\alpha}, D_{0+}^{\beta}$ and $D_{0+}^{\gamma}$ are the standard Riemann-Liouville fractional derivatives with $1<\alpha \leq 2,0<\beta, \gamma \leq 1,0 \leq \alpha-\beta-1, \lambda \in(0,+\infty), 0<\xi_{i}, \eta_{i}<1, i=1,2, \ldots, m-2$, $\sum_{i=1}^{m-2} \xi_{i} \eta_{i}^{\alpha-\beta-1}<1,0 \leq \alpha-\gamma-1, f \in C([0,1] \times[0,+\infty),[0,+\infty))$, and $\varphi_{p}(s)=|s|^{p-2} s, p>1$, $\varphi_{p}^{-1}=\varphi_{q}, \frac{1}{p}+\frac{1}{q}=1$.

Our work presented in this paper has the following features. Firstly, to the best of the author's knowledge, there are few results on the existence of solutions for nonlinear fractional $p$-Laplacian differential equations with $\mathrm{m}$-point boundary value problems. Secondly, we transform (1.1) into an equivalent integral equation and discuss the eigenvalue interval for the existence of multiplicity of positive solutions. The paper is organized as follows. In Section 2, we present some background materials and preliminaries. Section 3 deals with some existence results. In Section 4, two examples are given to illustrate the results.

\section{Background materials and preliminaries}

Definition $2.1([10,11])$ The fractional integral of order $\alpha$ with the lower limit $t_{0}$ for a function $f$ is defined as

$$
I^{\alpha} f(t)=\frac{1}{\Gamma(\alpha)} \int_{t_{0}}^{t}(t-s)^{\alpha-1} f(s) d s, \quad t>t_{0}, \alpha>0,
$$

where $\Gamma$ is the gamma function.

Definition 2.2 ([10,11]) The Riemann-Liouville derivative of order $\alpha$ with the lower limit $t_{0}$ for a function $f$ is

$$
D_{t_{0}}^{\alpha} f(t)=\frac{1}{\Gamma(n-\alpha)}\left(\frac{d}{d t}\right)^{n} \int_{t_{0}}^{t}(t-s)^{n-\alpha-1} f(s) d s, \quad t>t_{0}, \alpha>0, n=[\alpha]+1 .
$$

Lemma 2.1 ([12]) Assume that $u \in C(0,1) \cap L^{1}(0,1)$ with a fractional derivative of order $\alpha>0$ that belongs to $C(0,1) \cap L^{1}(0,1)$. Then

$$
I_{0+}^{\alpha} D_{0+}^{\alpha} u(t)=u(t)+C_{1} t^{\alpha-1}+C_{2} t^{\alpha-2}+\cdots+C_{N} t^{\alpha-N} \quad \text { for some } C_{i} \in \mathbb{R}, i=1,2, \ldots, N,
$$

where $N$ is the smallest integer greater than or equal to $\alpha$.

Lemma 2.2 ([7]) Let $y \in C[0,1]$. Then the fractional differential equation

$$
\left\{\begin{array}{l}
D_{0+}^{\alpha} u(t)+y(t)=0, \quad 0<t<1,1<\alpha \leq 2 \\
u(0)=0, \quad D_{0+}^{\beta} u(1)=\sum_{i=1}^{m-2} \xi_{i} D_{0+}^{\beta} u\left(\eta_{i}\right)
\end{array}\right.
$$


has a unique solution which is given by

$$
u(t)=\int_{0}^{1} G(t, s) y(s) d s
$$

where

$$
G(t, s)=G_{1}(t, s)+G_{2}(t, s)
$$

in which

$$
\begin{aligned}
& G_{1}(t, s)= \begin{cases}\frac{t^{\alpha-1}(1-s)^{\alpha-\beta-1}-(t-s)^{\alpha-1}}{\Gamma(\alpha)}, & 0 \leq s \leq t \leq 1, \\
\frac{t^{\alpha-1}(1-s)^{\alpha-\beta-1}}{\Gamma(\alpha)}, & 0 \leq t \leq s \leq 1,\end{cases} \\
& G_{2}(t, s)= \begin{cases}\frac{1}{A \Gamma(\alpha)}\left[\sum_{0 \leq s \leq \eta_{i}}\left(\xi_{i} \eta_{i}^{\alpha-\beta-1} t^{\alpha-1}(1-s)^{\alpha-\beta-1}-\xi_{i} t^{\alpha-1}\left(\eta_{i}-s\right)^{\alpha-\beta-1}\right)\right], & t \in[0,1], \\
\frac{1}{A \Gamma(\alpha)}\left(\sum_{\eta_{i} \leq s \leq 1} \xi_{i} \eta_{i}{ }^{\alpha-\beta-1} t^{\alpha-1}(1-s)^{\alpha-\beta-1}\right), & t \in[0,1],\end{cases}
\end{aligned}
$$

where

$$
A=1-\sum_{i=1}^{m-2} \xi_{i} \eta_{i}^{\alpha-\beta-1}
$$

Lemma 2.3 ([7]) If $\sum_{i=1}^{m-2} \xi_{i} \eta_{i}^{\alpha-\beta-1}<1$, then the function $G(t, s)$ in Lemma 2.2 satisfies the following conditions:

(i) $G(t, s)>0$, for $s, t \in(0,1)$,

(ii) $G(t, s) \leq G_{*}(s, s)$, for $s, t \in[0,1]$,

where

$$
G_{*}(s, s)=\frac{1}{\Gamma(\alpha)}(1-s)^{\alpha-\beta-1}+\frac{1}{A \Gamma(\alpha)} \sum_{i=1}^{m-2} \xi_{i} \eta_{i}^{\alpha-\beta-1}(1-s)^{\alpha-\beta-1} .
$$

Lemma 2.4 $G(t, s)$ in [7] has the following property:

$$
G(t, s) \geq t^{\alpha-1} G(1, s) .
$$

Proof For $0 \leq s \leq t \leq 1$, we conclude that

$$
\begin{aligned}
& t^{\alpha-1}(1-s)^{\alpha-\beta-1}-(t-s)^{\alpha-1} \\
& \quad=t^{\alpha-1}\left[(1-s)^{\alpha-\beta-1}-\left(1-\frac{s}{t}\right)^{\alpha-1}\right] \\
& \quad \geq t^{\alpha-1}\left[(1-s)^{\alpha-\beta-1}-(1-s)^{\alpha-1}\right] .
\end{aligned}
$$

Thus

$$
G_{1}(t, s) \geq t^{\alpha-1} G_{1}(1, s) .
$$

It is obvious that

$$
G_{2}(t, s) \geq t^{\alpha-1} G_{2}(1, s) .
$$


Therefore

$$
G(t, s) \geq t^{\alpha-1} G(1, s) .
$$

Lemma 2.5 Let $f \in C([0,1] \times[0,+\infty),[0,+\infty))$, then $B V P(1.1)$ has a unique solution

$$
u(t)=\lambda \int_{0}^{1} G(t, s) \varphi_{p}^{-1}\left(I_{0+}^{\beta} f(s, u(s))\right) d s
$$

Proof Let $w=D_{0+}^{\alpha} u, v=\varphi_{p}(w)$. From (1.1), we have

$$
\left\{\begin{array}{l}
D_{0+}^{\beta} v(t)+\varphi_{p}(\lambda) f(t, u(t))=0, \quad 0<t<1 \\
v(0)=0
\end{array}\right.
$$

By Lemma 2.1, we have

$$
v(t)=c_{1} t^{\beta-1}-I_{0+}^{\beta}\left(\varphi_{p}(\lambda) f(t, u(t))\right), \quad 0<t<1 .
$$

It follows from $v(0)=0$ that

$$
v(t)=-I_{0+}^{\beta}\left(\varphi_{p}(\lambda) f(t, u(t))\right), \quad 0<t<1 .
$$

Thus, from (1.1) we know that

$$
\left\{\begin{array}{l}
D_{0+}^{\alpha} u(t)=\varphi_{p}^{-1}\left(-I_{0+}^{\beta}\left(\varphi_{p}(\lambda) f(t, u(t))\right)\right), \quad 0<t<1 \\
u(0)=0, \quad D_{0+}^{\gamma} u(1)=\sum_{i=1}^{m-2} \xi_{i} D_{0+}^{\gamma} u\left(\eta_{i}\right)
\end{array}\right.
$$

By Lemma 2.2, (1.1) has a unique solution

$$
u(t)=-\lambda \int_{0}^{1} G(t, s) \varphi_{p}^{-1}\left(-I_{0+}^{\beta} f(s, u(s))\right) d s .
$$

It follows from $f \in C([0,1] \times[0,+\infty),[0,+\infty))$ that

$$
-\int_{0}^{1} G(t, s) \varphi_{p}^{-1}\left(-I_{0+}^{\beta} f(s, u(s))\right) d s=\int_{0}^{1} G(t, s) \varphi_{p}^{-1}\left(I_{0+}^{\beta} f(s, u(s))\right) d s .
$$

Thus

$$
u(t)=\lambda \int_{0}^{1} G(t, s) \varphi_{p}^{-1}\left(I_{0+}^{\beta} f(s, u(s))\right) d s .
$$

Lemma 2.6 ([27]) Let E be a real Banach space, $P \subset E$ be a cone, $\Omega_{r}=\{u \in P:\|u\| \leq r\}$. Let the operator $T: P \cap \Omega_{r} \rightarrow P$ be completely continuous and satisfy $T x \neq x, \forall x \in \partial \Omega_{r}$. Then

(i) If $\|T x\| \leq\|x\|, \quad \forall x \in \partial \Omega_{r}, \quad$ then $i\left(T, \Omega_{r}, P\right)=1$,

(ii) If $\|T x\| \geq\|x\|, \quad \forall x \in \partial \Omega_{r}$, then $i\left(T, \Omega_{r}, P\right)=0$. 


\section{Main results}

We consider the Banach space $E=C([0,1], \mathbb{R})$ endowed with the norm defined by $\|u\|=$ $\sup _{0 \leq t \leq 1}|u(t)|$. Let $P=\{u \in E \mid u(t) \geq 0\}$, then $P$ is a cone in $E$. Define an operator $T: P \rightarrow P$

as

$$
(T u)(t)=\lambda \int_{0}^{1} G(t, s) \varphi_{p}^{-1}\left(I_{0+}^{\beta} f(s, u(s))\right) d s
$$

Then $T$ has a solution if and only if the operator $T$ has a fixed point.

Lemma 3.1 If $f \in C([0,1] \times[0,+\infty),[0,+\infty))$, then the operator $T: P \rightarrow P$ is completely continuous.

Proof From the continuity and non-negativeness of $G(t, s)$ and $f(t, u(t))$, we know that $T: P \rightarrow P$ is continuous.

Let $\Omega \subset P$ be bounded. Then, for all $t \in[0,1]$ and $u \in \Omega$, there exists a positive constant $M$ such that $|f(t, u(t))| \leq M$. Thus,

$$
\begin{aligned}
|(T u)(t)| & =\left|\lambda \int_{0}^{1} G(t, s) \varphi_{p}^{-1}\left(I_{0+}^{\beta} f(s, u(s))\right) d s\right| \\
& \leq \lambda \int_{0}^{1} G_{*}(s, s)\left(\int_{0}^{s}(s-\tau)^{\beta-1} d \tau\right)^{q-1} d s \frac{M^{q-1}}{(\Gamma(\beta))^{q-1}} \\
& =\lambda \frac{M^{q-1}}{(\Gamma(\beta+1))^{q-1}} \int_{0}^{1} G_{*}(s, s) s^{(q-1) \beta} d s \\
& \leq \lambda \frac{M^{q-1}}{(\Gamma(\beta+1))^{q-1}} \int_{0}^{1} G_{*}(s, s) d s \\
& =\lambda \frac{M^{q-1} L}{(\Gamma(\beta+1))^{q-1}},
\end{aligned}
$$

where

$$
L=\int_{0}^{1} G_{*}(s, s) d s
$$

This means that $T(\Omega)$ is uniformly bounded.

On the other hand, from the continuity of $G(t, s)$ on $[0,1] \times[0,1]$, we see that it is uniformly continuous on $[0,1] \times[0,1]$. Thus, for fixed $s \in[0,1]$ and for any $\varepsilon>0$, there exists a constant $\delta>0$ such that $t_{1}, t_{2} \in[0,1]$ and $\left|t_{1}-t_{2}\right|<\delta$,

$$
\left|G\left(t_{1}, s\right)-G\left(t_{2}, s\right)\right|<\frac{(\Gamma(\beta+1))^{q-1}}{\lambda M^{q-1}} \varepsilon
$$

Hence, for all $u \in \Omega$,

$$
\begin{aligned}
& \left|(T u)\left(t_{2}\right)-(T u)\left(t_{1}\right)\right| \\
& \quad \leq \lambda \int_{0}^{1}\left|G\left(t_{2}, s\right)-G\left(t_{1}, s\right)\right| \varphi_{p}^{-1}\left(I_{0+}^{\beta} f(s, u(s))\right) d s
\end{aligned}
$$


Lv Advances in Difference Equations 2014, 2014:69

Page 7 of 16

http://www.advancesindifferenceequations.com/content/2014/1/69

$$
\begin{aligned}
& \leq \lambda \int_{0}^{1}\left|G\left(t_{2}, s\right)-G\left(t_{1}, s\right)\right|\left(\int_{0}^{s}(s-\tau)^{\beta-1} d \tau\right)^{q-1} d s \frac{M^{q-1}}{(\Gamma(\beta))^{q-1}} \\
& =\lambda \frac{M^{q-1}}{(\Gamma(\beta+1))^{q-1}} \int_{0}^{1}\left|G\left(t_{2}, s\right)-G\left(t_{1}, s\right)\right| s^{(q-1) \beta} d s \\
& \leq \lambda \frac{M^{q-1}}{(\Gamma(\beta+1))^{q-1}} \int_{0}^{1}\left|G\left(t_{2}, s\right)-G\left(t_{1}, s\right)\right| d s \\
& =\varepsilon
\end{aligned}
$$

which implies that $T(\Omega)$ is equicontinuous. By the Arzela-Ascoli theorem, we obtain that $T: P \rightarrow P$ is completely continuous. The proof is complete.

Theorem 3.2 If $f \in C([0,1] \times[0,+\infty),[0,+\infty)), f(t, u)$ is nondecreasing in $u$ and $\lambda \in$ $(0,+\infty)$, then $B V P(1.1)$ has a minimal positive solution $\bar{v}$ in $B_{r}$ and a maximal positive solution $\bar{w}$ in $B_{r}$. Moreover, $v_{m}(t) \rightarrow \bar{v}(t), w_{m}(t) \rightarrow \bar{w}(t)$ as $m \rightarrow \infty$ uniformly on [0,1], where

$$
v_{m}(t)=\lambda \int_{0}^{1} G(t, s) \varphi_{p}^{-1}\left(I_{0+}^{\beta} f\left(s, v_{m-1}(s)\right)\right) d s
$$

and

$$
w_{m}(t)=\lambda \int_{0}^{1} G(t, s) \varphi_{p}^{-1}\left(I_{0+}^{\beta} f\left(s, w_{m-1}(s)\right)\right) d s .
$$

Proof Let

$$
B_{r}=\{u \in P:\|u\| \leq r\}
$$

where

$$
r \geq \frac{\lambda M_{1}^{q-1}}{(\Gamma(\beta+1))^{q-1}} \int_{0}^{1} G_{*}(s, s) d s .
$$

Step 1: Problem (1.1) has at least one solution.

For $u \in B_{r}$, there exists a positive constant $M_{1}$ such that $|f(t, u(t))| \leq M_{1}$,

$$
\begin{aligned}
|(T u)(t)| & =\left|\lambda \int_{0}^{1} G(t, s) \varphi_{p}^{-1}\left(I_{0+}^{\beta} f(s, u(s))\right) d s\right| \\
& \leq \frac{\lambda}{(\Gamma(\beta))^{q-1}} \int_{0}^{1} G_{*}(s, s)\left(\int_{0}^{s}(s-\tau)^{\beta-1} f(\tau, u(\tau)) d \tau\right)^{q-1} d s \\
& \leq \frac{\lambda M_{1}^{q-1}}{(\Gamma(\beta))^{q-1}} \int_{0}^{1} G_{*}(s, s)\left(\int_{0}^{s}(s-\tau)^{\beta-1} d \tau\right)^{q-1} d s \\
& =\frac{\lambda M_{1}^{q-1}}{(\Gamma(\beta+1))^{q-1}} \int_{0}^{1} G_{*}(s, s) s^{\beta(q-1)} d s \\
& \leq \frac{\lambda M_{1}^{q-1}}{(\Gamma(\beta+1))^{q-1}} \int_{0}^{1} G_{*}(s, s) d s .
\end{aligned}
$$


Thus

$$
T: B_{r} \rightarrow B_{r}
$$

By Lemma 3.1, we can see that $T: B_{r} \rightarrow B_{r}$ is completely continuous. Hence, by means of the Schauder fixed point theorem, the operator $T$ has at least one fixed point, and BVP (1.1) has at least one solution in $B_{r}$.

Step 2: BVP (1.1) has a positive solution in $B_{r}$, which is a minimal positive solution.

From (3.1) and (3.2), one can see that

$$
v_{m}(t)=\left(T v_{m-1}\right)(t), \quad t \in[0,1], m=1,2,3, \ldots
$$

This, together with $f(t, u)$ being nondecreasing in $u$, yields that

$$
0=v_{0}(t) \leq v_{1}(t) \leq \cdots \leq v_{m}(t) \leq \cdots, \quad t \in[0,1]
$$

Since $T$ is compact, we obtain that $\left\{v_{m}\right\}$ is a sequentially compact set. Consequently, there exists $\bar{v} \in B_{r}$ such that $v_{m} \rightarrow \bar{v}(m \rightarrow \infty)$.

Let $u(t)$ be any positive solution of BVP (1.1) in $B_{r}$. It is obvious that $0=v_{0}(t) \leq u(t)=$ $(T u)(t)$.

Thus,

$$
v_{m}(t) \leq u(t) \quad(m=0,1,2,3, \ldots) .
$$

Taking limits as $m \rightarrow \infty$ in (3.5), we get $\bar{v}(t) \leq u(t)$ for $t \in[0,1]$.

Step 3: BVP (1.1) has a positive solution in $B_{r}$, which is a maximal positive solution. Let $w_{0}(t)=r, t \in[0,1]$ and $w_{1}(t)=T w_{0}(t)$. From $T: B_{r} \rightarrow B_{r}$, we have $w_{1} \in B_{r}$. Thus

$$
0 \leq w_{1}(t) \leq r=w_{0}(t)
$$

This, together with $f(t, u)$ being nondecreasing in $u$, yields that

$$
\cdots \leq w_{m}(t) \leq \cdots \leq w_{1}(t) \leq w_{0}(t), \quad t \in[0,1]
$$

Using a proof similar to that of Step 2, we can show that

$$
w_{m}(t) \rightarrow \bar{w}(t) \quad(m \rightarrow \infty)
$$

and

$$
\bar{w}(t)=\int_{0}^{1} G(t, s) f(s, \bar{w}(s)) d s .
$$

Let $u(t)$ be any positive solution of BVP (1.1) in $B_{r}$.

Obviously,

$$
u(t) \leq w_{0}(t)
$$


Thus

$$
u(t) \leq w_{m}(t)
$$

Taking limits as $m \rightarrow \infty$ in (3.6), we obtain $u(t) \leq \bar{w}(t)$ for $t \in[0,1]$.

The proof is complete.

Define

$$
\begin{aligned}
& f^{0}=\lim _{u \rightarrow 0+} \sup _{t \in[0,1]} \frac{f(t, u)}{\varphi_{p}\left(l_{1}\|u\|\right)}, \quad f_{0}=\lim _{u \rightarrow 0+} \inf _{t \in[0,1]} \frac{f(t, u)}{\varphi_{p}\left(l_{2}\|u\|\right)}, \\
& f^{\infty}=\lim _{u \rightarrow+\infty} \sup _{t \in[0,1]} \frac{f(t, u)}{\varphi_{p}\left(l_{3}\|u\|\right)}, \quad f_{\infty}=\lim _{u \rightarrow+\infty} \inf _{t \in[0,1]} \frac{f(t, u)}{\varphi_{p}\left(l_{4}\|u\|\right)} .
\end{aligned}
$$

Let

$$
B=\int_{0}^{1} G_{*}(s, s) s^{\beta(q-1)} d s \quad \text { and } \quad B_{1}=\int_{0}^{1} G(1, s) s^{\beta(q-1)} d s .
$$

Theorem 3.3 Assume that $f \in C([0,1] \times[0,+\infty),[0,+\infty))$, and the following conditions hold:

$\left(\mathrm{H}_{1}\right) f_{0}=f_{\infty}=+\infty$.

$\left(\mathrm{H}_{2}\right)$ There exists a constant $\rho_{1}>0$ such that $f(t, u) \leq \varphi_{p}\left(l_{5}\|u\|\right)$ for $t \in[0,1], u \in\left[0, \rho_{1}\right]$.

Then BVP (1.1) has at least two positive solutions $u_{1}$ and $u_{2}$ such that

$$
0<\left\|u_{1}\right\|<\rho_{1}<\left\|u_{2}\right\|
$$

for

$$
\lambda \in\left(\frac{(\Gamma(\beta+1))^{q-1}}{l_{2} B_{1}}, \frac{(\Gamma(\beta+1))^{q-1}}{l_{5} B}\right) \cap\left(\frac{(\Gamma(\beta+1))^{q-1}}{l_{4} B_{1}}, \frac{(\Gamma(\beta+1))^{q-1}}{l_{5} B}\right),
$$

where

$$
l_{2} B_{1}>l_{5} B \text { and } l_{4} B_{1}>l_{5} B .
$$

Proof Since

$$
f_{0}=\lim _{u \rightarrow 0+} \inf _{t \in[0,1]} \frac{f(t, u)}{\varphi_{p}\left(l_{2}\|u\|\right)}=+\infty
$$

there is $\rho_{0} \in\left(0, \rho_{1}\right)$ such that

$$
f(t, u) \geq \varphi_{p}\left(l_{2}\|u\|\right) \quad \text { for } t \in[0,1], u \in\left[0, \rho_{0}\right] .
$$

Let

$$
\Omega_{\rho_{0}}=\left\{u \in P:\|u\| \leq \rho_{0}\right\} .
$$


Then, for any $u \in \partial \Omega_{\rho_{0}}$, it follows from Lemma 2.4 that

$$
\begin{aligned}
(T u)(t) & =\lambda \int_{0}^{1} G(t, s) \varphi_{p}^{-1}\left(I_{0+}^{\beta} f(s, u(s))\right) d s \\
& \geq \lambda \int_{0}^{1} t^{\alpha-1} G(1, s) \varphi_{p}^{-1}\left(I_{0+}^{\beta}\left(\varphi_{p}\left(l_{2}\|u\|\right)\right)\right) d s \\
& =\lambda l_{2} \int_{0}^{1} t^{\alpha-1} G(1, s)\left(\frac{1}{\Gamma(\beta)} \int_{0}^{s}(s-\tau)^{\beta-1} d \tau\right)^{q-1} d s\|u\| \\
& =\frac{\lambda l_{2}}{(\Gamma(\beta+1))^{q-1}} \int_{0}^{1} t^{\alpha-1} G(1, s) s^{\beta(q-1)} d s\|u\| .
\end{aligned}
$$

Thus

$$
\|T u\| \geq \frac{\lambda l_{2} B_{1}}{(\Gamma(\beta+1))^{q-1}}\|u\| .
$$

This, together with (3.7), yields that

$$
\|T u\| \geq\|u\|, \quad \forall u \in \partial \Omega_{\rho_{0}} .
$$

By Lemma 2.6, we have

$$
i\left(T, \Omega_{\rho_{0}}, P\right)=0 \text {. }
$$

In view of

$$
f_{\infty}=\lim _{u \rightarrow+\infty} \inf _{t \in[0,1]} \frac{f(t, u)}{\varphi_{p}\left(l_{4}\|u\|\right)}=+\infty
$$

there is $\rho_{0}^{*}, \rho_{0}^{*}>\rho_{1}$, such that

$$
f(t, u) \geq \varphi_{p}\left(l_{4}\|u\|\right) \quad \text { for } t \in[0,1], u \in\left[\rho_{0}^{*},+\infty\right) .
$$

Let

$$
\Omega_{\rho_{0}^{*}}=\left\{u \in P:\|u\| \leq \rho_{0}^{*}\right\}
$$

Then, for any $u \in \partial \Omega_{\rho_{0}^{*}}$, it follows from Lemma 2.4 that

$$
\begin{aligned}
(T u)(t) & =\lambda \int_{0}^{1} G(t, s) \varphi_{p}^{-1}\left(I_{0+}^{\beta} f(s, u(s))\right) d s \\
& \geq \lambda \int_{0}^{1} t^{\alpha-1} G(1, s) \varphi_{p}^{-1}\left(I_{0+}^{\beta}\left(\varphi_{p}\left(l_{4}\|u\|\right)\right)\right) d s \\
& =\lambda l_{4} \int_{0}^{1} t^{\alpha-1} G(1, s)\left(\frac{1}{\Gamma(\beta)} \int_{0}^{s}(s-\tau)^{\beta-1} d \tau\right)^{q-1} d s\|u\| \\
& =\frac{\lambda l_{4}}{(\Gamma(\beta+1))^{q-1}} \int_{0}^{1} t^{\alpha-1} G(1, s) s^{\beta(q-1)} d s\|u\| .
\end{aligned}
$$


Thus

$$
\|T u\| \geq \frac{\lambda l_{4} B_{1}}{(\Gamma(\beta+1))^{q-1}}\|u\| .
$$

This, together with (3.7), yields that

$$
\|T u\| \geq\|u\|, \quad \forall u \in \partial \Omega_{\rho_{0}^{*}} .
$$

By Lemma 2.6, we have

$$
i\left(T, \Omega_{\rho_{0}^{*}}, P\right)=0 \text {. }
$$

Finally, let $\Omega_{\rho_{1}}=\left\{u \in P:\|u\| \leq \rho_{1}\right\}$. For any $u \in \partial \Omega_{\rho_{1}}$, it follows from Lemma 2.3 and $\left(\mathrm{H}_{2}\right)$ that

$$
\begin{aligned}
(T u)(t) & =\lambda \int_{0}^{1} G(t, s) \varphi_{p}^{-1}\left(I_{0+}^{\beta} f(s, u(s))\right) d s \\
& \leq \lambda \int_{0}^{1} G_{*}(s, s) \varphi_{p}^{-1}\left(I_{0+}^{\beta}\left(\varphi_{p}\left(l_{5}\|u\|\right)\right)\right) d s \\
& =\lambda l_{5} \int_{0}^{1} G_{*}(s, s)\left(\frac{1}{\Gamma(\beta)} \int_{0}^{s}(s-\tau)^{\beta-1} d \tau\right)^{q-1} d s\|u\| \\
& =\frac{\lambda l_{5}}{(\Gamma(\beta+1))^{q-1}} \int_{0}^{1} G_{*}(s, s) s^{\beta(q-1)} d s\|u\| .
\end{aligned}
$$

Thus

$$
\|T u\| \leq \frac{\lambda l_{5} B}{(\Gamma(\beta+1))^{q-1}}\|u\| .
$$

This, together with (3.7), yields that

$$
\|T u\| \leq\|u\|, \quad \forall u \in \partial \Omega_{\rho_{1}} .
$$

Using Lemma 2.6, we get

$$
i\left(T, \Omega_{\rho_{1}}, P\right)=1 \text {. }
$$

From (3.8)-(3.10) and $\rho_{0}<\rho_{1}<\rho_{0}^{*}$, we have

$$
i\left(T, \Omega_{\rho_{0}^{*}} \backslash \bar{\Omega}_{\rho_{1}}, P\right)=-1, \quad i\left(T, \Omega_{\rho_{1}} \backslash \bar{\Omega}_{\rho_{0}}, P\right)=1 .
$$

Therefore, $T$ has a fixed point $u_{1} \in \Omega_{\rho_{1}} \backslash \bar{\Omega}_{\rho_{0}}$ and a fixed point $u_{2} \in \Omega_{\rho_{0}^{*}} \backslash \bar{\Omega}_{\rho_{1}}$. Clearly, $u_{1}, u_{2}$ are both positive solutions of BVP (1.1) and $0<\left\|u_{1}\right\|<\rho_{1}<\left\|u_{2}\right\|$. The proof of Theorem 3.3 is completed.

In a similar way, we can obtain the following result. 
Corollary 3.4 Assume that $f \in C([0,1] \times[0,+\infty),[0,+\infty))$, and the following conditions hold:

$\left(\mathrm{H}_{1}\right) f^{0}=f^{\infty}=0$

$\left(\mathrm{H}_{2}\right)$ There exists a constant $\rho_{2}>0$ such that $f(t, u) \geq \varphi_{p}\left(l_{6}\|u\|\right)$ for $t \in[0,1], u \in\left[0, \rho_{2}\right]$.

Then BVP (1.1) has at least two positive solutions $u_{1}$ and $u_{2}$ such that

$$
0<\left\|u_{1}\right\|<\rho_{2}<\left\|u_{2}\right\|
$$

for

$$
\lambda \in\left(\frac{(\Gamma(\beta+1))^{q-1}}{l_{6} B_{1}}, \frac{(\Gamma(\beta+1))^{q-1}}{l_{3} B}\right) \cap\left(\frac{(\Gamma(\beta+1))^{q-1}}{l_{6} B_{1}}, \frac{(\Gamma(\beta+1))^{q-1}}{l_{1} B}\right),
$$

where

$$
l_{6} B_{1}>l_{3} B \text { and } l_{6} B_{1}>l_{1} B .
$$

\section{Examples}

Example 4.1 Consider the following boundary value problem:

$$
\begin{cases}D_{0_{+}}^{\frac{1}{2}}\left(\varphi_{2}\left(D_{0_{+}}^{\frac{3}{2}} u(t)\right)\right)+\varphi_{2}(\lambda)\left((t+1) \pi \frac{|u(t)|}{1+|u(t)|}\right)=0, & 0<t<1, \\ u(0)=0, \quad D_{0_{+}}^{\frac{1}{2}} u(1)=\sum_{i=1}^{2} \xi_{i} D_{0+}^{\frac{1}{2}} u\left(\eta_{i}\right), & D_{0+}^{\frac{3}{2}} u(0)=0,\end{cases}
$$

where

$$
\begin{aligned}
& \alpha=\frac{3}{2}, \quad \beta=\frac{1}{2}, \quad \gamma=\frac{1}{2}, \quad m=4, \quad p=q=2, \\
& \xi_{1}=\eta_{1}=\frac{1}{4}, \quad \xi_{2}=\eta_{2}=\frac{1}{2}, \quad \lambda \in(0,+\infty), \quad f(t, u)=(t+1) \pi \frac{|u(t)|}{1+|u(t)|} .
\end{aligned}
$$

Thus

$$
f \in C([0,1] \times[0,+\infty),[0,+\infty)) \text { and }|f(t, u)|=\left|(t+1) \pi \frac{|u(t)|}{1+|u(t)|}\right| \leq 2 \pi
$$

By computation, we deduce that

$$
\begin{aligned}
& \sum_{i=1}^{2} \xi_{i} \eta_{i}^{\alpha-\beta-1}=\xi_{i}+\xi_{2}=\frac{3}{4}, \\
& A=1-\sum_{i=1}^{2} \xi_{i} \eta_{i}^{\alpha-\beta-1}=\frac{1}{4}
\end{aligned}
$$

and

$$
\alpha-\gamma-1 \geq 0 .
$$


On the other hand,

$$
\begin{aligned}
\int_{0}^{1} G_{*}(s, s) d s & =\frac{1}{\Gamma(\alpha)} \int_{0}^{1}(1-s)^{\alpha-\beta-1} d s+\frac{1}{A \Gamma(\alpha)} \sum_{i=1}^{2} \xi_{i} \eta_{i}^{\alpha-\beta-1} \int_{0}^{1}(1-s)^{\alpha-\beta-1} d s \\
& =\left(\frac{1}{\Gamma(\alpha)}+\frac{1}{A \Gamma(\alpha)} \sum_{i=1}^{2} \xi_{i} \eta_{i}^{\alpha-\beta-1}\right) \int_{0}^{1}(1-s)^{\alpha-\beta-1} d s \\
& =\frac{1}{\Gamma(\alpha)}+\frac{1}{A \Gamma(\alpha)} \sum_{i=1}^{2} \xi_{i} \\
& =\frac{1}{\frac{\sqrt{\pi}}{2}}+\frac{1}{\frac{1}{4} \frac{\sqrt{\pi}}{2}} \frac{3}{4} \\
& =\frac{2}{\sqrt{\pi}}+\frac{6}{\sqrt{\pi}} \\
& =\frac{8}{\sqrt{\pi}} .
\end{aligned}
$$

Take

$$
\begin{aligned}
r & \geq \frac{\lambda M_{1}^{q-1}}{(\Gamma(\beta+1))^{q-1}} \int_{0}^{1} G_{*}(s, s) d s \\
& =\frac{\lambda 2 \pi}{\frac{\sqrt{\pi}}{2}} \frac{8}{\sqrt{\pi}} \\
& =32 \lambda .
\end{aligned}
$$

Hence, by Theorem 3.2, BVP (4.1) has a minimal positive solution $\bar{v}$ in $B_{r}$ and a maximal positive solution $\bar{w}$ in $B_{r}$.

Example 4.2 Consider the following boundary value problem:

$$
\left\{\begin{array}{l}
D_{0+}^{\frac{1}{2}}\left(\varphi_{\frac{3}{2}}\left(D_{0+}^{\frac{3}{2}} u(t)\right)\right)+\varphi_{\frac{3}{2}}(\lambda)(1+t)\left(\frac{1}{2}|u(t)|^{\frac{1}{3}}+\frac{1}{2}\|u\|^{\frac{1}{3}}+\|u\|^{2}\right)=0, \quad 0<t<1, \\
u(0)=0, \quad D_{0+}^{\frac{1}{2}} u(1)=\sum_{i=1}^{2} \xi_{i} D_{0+}^{\frac{1}{2}} u\left(\eta_{i}\right), \quad D_{0+}^{\frac{3}{2}} u(0)=0,
\end{array}\right.
$$

where

$$
\begin{aligned}
& \alpha=\frac{3}{2}, \quad \beta=\frac{1}{2}, \quad \gamma=\frac{1}{2}, \quad p=\frac{3}{2}, \quad q=3, \quad m=4, \\
& \xi_{1}=\eta_{1}=\frac{1}{4}, \quad \xi_{2}=\eta_{2}=\frac{1}{2}, \quad \alpha-\gamma-1=0, \quad \alpha-\beta-1=0
\end{aligned}
$$

and

$$
f(t, u)=(1+t)\left(\frac{1}{2}|u(t)|^{\frac{1}{3}}+\frac{1}{2}\|u\|^{\frac{1}{3}}+\|u\|^{2}\right)
$$

It follows from Example 4.1 that

$$
\sum_{i=1}^{2} \xi_{i} \eta_{i}^{\alpha-\beta-1}=\xi_{i}+\xi_{2}=\frac{3}{4}, \quad A=1-\sum_{i=1}^{2} \xi_{i} \eta_{i}^{\alpha-\beta-1}=\frac{1}{4}
$$


Lv Advances in Difference Equations 2014, 2014:69

Page 14 of 16

http://www.advancesindifferenceequations.com/content/2014/1/69

By computation, we deduce that

$$
\begin{aligned}
B & =\int_{0}^{1} G_{*}(s, s) s^{\beta(q-1)} d s \\
& =\frac{1}{\Gamma(\alpha)} \int_{0}^{1}(1-s)^{\alpha-\beta-1} s^{\beta(q-1)} d s+\frac{1}{A \Gamma(\alpha)} \sum_{i=1}^{2} \xi_{i} \eta_{i}^{\alpha-\beta-1} \int_{0}^{1}(1-s)^{\alpha-\beta-1} s^{\beta(q-1)} d s \\
& =\frac{1}{\Gamma(\alpha)} \int_{0}^{1} s^{\frac{1}{2} \times(3-1)} d s+\frac{1}{A \Gamma(\alpha)} \sum_{i=1}^{2} \xi_{i} \int_{0}^{1} s^{\frac{1}{2} \times(3-1)} d s \\
& =\frac{1}{\Gamma(\alpha)} \int_{0}^{1} s d s+\frac{1}{A \Gamma(\alpha)} \sum_{i=1}^{2} \xi_{i} \int_{0}^{1} s d s \\
& =\frac{1}{2}\left(\frac{1}{\Gamma(\alpha)}+\frac{1}{A \Gamma(\alpha)} \sum_{i=1}^{2} \xi_{i}\right) \\
& =\frac{1}{2}\left(\frac{2}{\sqrt{\pi}}+\frac{8}{\sqrt{\pi}}\left(\frac{1}{2}+\frac{1}{4}\right)\right) \\
& =\frac{1}{2} \times \frac{8}{\sqrt{\pi}} \\
& =\frac{4}{\sqrt{\pi}}
\end{aligned}
$$

and

$$
\begin{aligned}
B_{1}= & \int_{0}^{1} G(1, s) s^{\beta(q-1)} d s \\
= & \int_{0}^{1} G_{1}(1, s) s^{\beta(q-1)} d s+\int_{0}^{1} G_{2}(1, s) s^{\beta(q-1)} d s \\
= & \frac{1}{\Gamma\left(\frac{3}{2}\right)} \int_{0}^{1}\left[1-(1-s)^{\frac{1}{2}}\right] s^{\beta(q-1)} d s+\frac{1}{A \Gamma\left(\frac{3}{2}\right)} \int_{0}^{\frac{1}{4}}\left[\xi_{1} \eta_{1}^{0}(1-s)^{0}-\xi_{1}\left(\eta_{1}-s\right)^{0}\right] s^{\beta(q-1)} d s \\
& +\frac{1}{A \Gamma\left(\frac{3}{2}\right)} \int_{\frac{1}{4}}^{1} \xi_{1} \eta_{1}^{0}(1-s)^{0} s^{\beta(q-1)} d s \\
& +\frac{1}{A \Gamma\left(\frac{3}{2}\right)} \int_{0}^{\frac{1}{2}}\left[\xi_{2} \eta_{2}^{0}(1-s)^{0}-\xi_{2}\left(\eta_{2}-s\right)^{0}\right] s^{\beta(q-1)} d s \\
& +\frac{1}{A \Gamma\left(\frac{3}{2}\right)} \int_{\frac{1}{2}}^{1} \xi_{2} \eta_{2}^{0}(1-s)^{0} s^{\beta(q-1)} d s \\
= & \frac{1}{\Gamma\left(\frac{3}{2}\right)} \int_{0}^{1}\left[s-s(1-s)^{\frac{1}{2}}\right] d s+\frac{1}{A \Gamma\left(\frac{3}{2}\right)} \int_{\frac{1}{4}}^{1} \xi_{1} s d s+\frac{1}{A \Gamma\left(\frac{3}{2}\right)} \int_{\frac{1}{2}}^{1} \xi_{2} s d s \\
= & \frac{2}{\sqrt{\pi}} \int_{0}^{1}\left[s-s(1-s)^{\frac{1}{2}}\right] d s+\frac{2}{\sqrt{\pi}} \int_{\frac{1}{4}}^{1} s d s+\frac{4}{\sqrt{\pi}} \int_{\frac{1}{2}}^{1} s d s \\
= & \frac{2}{\sqrt{\pi}}\left(\frac{1}{2}-\frac{4}{15}\right)+\left.\frac{2}{\sqrt{\pi}} \frac{1}{2} s^{2}\right|_{\frac{1}{4}} ^{1}+\left.\frac{1}{\sqrt{\pi}} \frac{1}{2} s^{2}\right|_{\frac{1}{2}} ^{1} \\
& \\
& \frac{6}{\pi} \\
&
\end{aligned}
$$


Taking

$$
\rho_{1}=8, \quad l_{5}=2,178,
$$

we have

$$
f(t, u) \leq(1+1)(2+64)=132=\varphi_{p}\left(l_{5}\|u\|\right)=\varphi_{\frac{3}{2}}(2,178 \times 8) \quad \text { for } t \in[0,1], u \in\left[0, \rho_{1}\right]
$$

Thus, condition $\left(\mathrm{H}_{2}\right)$ is satisfied. It is obvious that condition $\left(\mathrm{H}_{1}\right)$ holds.

On the other hand, let $l_{2}=4,000, l_{4}=3,600$, we have $l_{2} B_{1}>l_{5} B, l_{4} B_{1}>l_{5} B$ and

$$
\begin{aligned}
\lambda & \in\left(\frac{(\Gamma(\beta+1))^{q-1}}{l_{2} B_{1}}, \frac{(\Gamma(\beta+1))^{q-1}}{l_{5} B}\right) \cap\left(\frac{(\Gamma(\beta+1))^{q-1}}{l_{4} B_{1}}, \frac{(\Gamma(\beta+1))^{q-1}}{l_{5} B}\right) \\
& =\left(\frac{(\Gamma(\beta+1))^{q-1}}{l_{4} B_{1}}, \frac{(\Gamma(\beta+1))^{q-1}}{l_{5} B}\right) \\
& =\left(\frac{\left(\frac{\sqrt{\pi}}{2}\right)^{2}}{3,600 \times \frac{697}{240 \sqrt{\pi}}}, \frac{\left(\frac{\sqrt{\pi}}{2}\right)^{2}}{2,178 \times \frac{4}{\sqrt{\pi}}}\right) \\
& =\left(\frac{\pi^{\frac{3}{2}}}{41,820}, \frac{\pi^{\frac{3}{2}}}{34,848}\right) .
\end{aligned}
$$

Hence, by Theorem 3.3, BVP (4.2) has at least two solutions $u_{1}$ and $u_{2}$ such that $0<\left\|u_{1}\right\|<$ $8<\left\|u_{2}\right\|$ for

$$
\lambda \in\left(\frac{\pi^{\frac{3}{2}}}{41,820}, \frac{\pi^{\frac{3}{2}}}{34,848}\right)
$$

\section{Competing interests}

The author declares that he has no competing interests.

\section{Acknowledgements}

This research is supported by Henan Province College Youth Backbone Teacher Funds (2011GGJS-213) and the National Natural Science Foundation of China (11271336).

Received: 21 November 2013 Accepted: 6 February 2014 Published: 18 Feb 2014

\section{References}

1. Wang, $J \mathrm{H}, \mathrm{Xiang}, \mathrm{HG}$ : Upper and lower solutions method for a class of singular fractional boundary value problems with p-Laplacian operator. Abstr. Appl. Anal. 2010, Article ID 971824 (2010)

2. Chai, GQ: Positive solutions for boundary value problem for fractional differential equation with $p$-Laplacian operator. Bound. Value Probl. 2012, 18 (2012)

3. Chen, TY, Liu, WB: A anti-periodic boundary value problem for the fractional differential equation with a $p$-Laplacian operator. Appl. Math. Lett. 25, 1671-1675 (2012)

4. Lu, HL, Han, ZL, Sun, SR, Liu, J: Existence on positive solutions for boundary value problems of nonlinear fractional differential equations with $p$-Laplacian. Adv. Differ. Equ. 2013, 30 (2013)

5. Bai, ZB: Eigenvalue intervals for a class of fractional boundary value problem. Comput. Math. Appl. 64, 3253-3257 (2012)

6. Zhang, XG, Liu, LS, Wu, YH: The eigenvalue problem for a singular higher order fractional differential equation involving fractional derivatives. Appl. Math. Comput. 218, 8526-8536 (2012)

7. $L v, Z W$ : Positive solutions of $m$-point boundary value problems for fractional differential equations. Adv. Differ. Equ. 2011, Article ID 571804 (2011)

8. LV, ZW, Liang, J, Xiao, TJ: Solutions to the Cauchy problem for differential equations in Banach spaces with fractional order. Comput. Math. Appl. 62, 1303-1311 (2011)

9. Lv, ZW, Liang, J, Xiao, TJ: Solutions to fractional differential equations with nonlocal initial condition in Banach spaces. Adv. Differ. Equ. 2010, Article ID 340349 (2010) 
10. Kilbas, AA, Srivastava, HM, Trujillo, JJ: Theory and Applications of Fractional Differential Equations. North-Holland Mathematics Studies, vol. 204. Elsevier, Amsterdam (2006)

11. Podlubny, I: Fractional Differential Equations. Academic Press, New York (1993)

12. Miller, KS, Ross, B: An Introduction to the Fractional Calculus and Fractional Differential Equations. Wiley, New York (1993)

13. Agarwal, RP, Belmekki, M, Benchohra, M: A survey on semilinear differential equations and inclusions involving Riemann-Liouville fractional derivative. Adv. Differ. Equ. 2009, Article ID 981728 (2009)

14. Agarwal, RP, Lakshmikantham, $\mathrm{V}$, Nieto, JJ: On the concept of solution for fractional differential equations with uncertainty. Nonlinear Anal. 72, 2859-2862 (2010)

15. Wang, RN, Xiao, TJ, Liang, J: A note on the fractional Cauchy problems with nonlocal initial conditions. Appl. Math. Lett. 24, 1435-1442 (2011)

16. Wang, RN, Chen, DH, Xiao, TJ: Abstract fractional Cauchy problems with almost sectorial operators. J. Differ. Equ. 252, 202-235 (2012)

17. Diagana, T, Mophou, GM, N'Guérékata, GM: On the existence of mild solutions to some semilinear fractional integro-differential equations. Electron. J. Qual. Theory Differ. Equ. 2010, 58 (2010)

18. Henderson, J, Ouahab, A: Fractional functional differential inclusions with finite delay. Nonlinear Anal. 70, 2091-2105 (2009)

19. Henderson, J, Ouahab, A: Impulsive differential inclusions with fractional order. Comput. Math. Appl. 59, 1191-1226 (2010)

20. Lakshmikantham, V: Theory of fractional differential equations. Nonlinear Anal. 60, 3337-3343 (2008)

21. Lakshmikantham, V, Vatsala, AS: Basic theory of fractional differential equations. Nonlinear Anal. 69, $2677-2682$ (2008)

22. Li, CF, Luo, XN, Zhou, Y: Existence of positive solutions of the boundary value problem for nonlinear fractional differential equations. Comput. Math. Appl. 59, 1363-1375 (2010)

23. Li, F: Mild solutions for fractional differential equations with nonlocal conditions. Adv. Differ. Equ. 2010, Article ID $287861(2010)$

24. Zhang, XQ: Positive solution for a class of singular semipositone fractional differential equations with integral boundary conditions. Bound. Value Probl. 2012, 123 (2012)

25. Mophou, GM: Existence and uniqueness of mild solutions to impulsive fractional differential equations. Nonlinear Anal. 72, 1604-1615 (2010)

26. Salem, $\mathrm{HAH}$ : On the fractional order m-point boundary value problem in reflexive Banach spaces and weak topologies. J. Comput. Appl. Math. 224, 565-572 (2009)

27. Guo, D, Lakshmikantham, V: Nonlinear Problems in Abstract Cone. Academic Press, San Diego (1998)

10.1186/1687-1847-2014-69

Cite this article as: LV: Existence results for m-point boundary value problems of nonlinear fractional differential equations with $p$-Laplacian operator. Advances in Difference Equations 2014, 2014:69

\section{Submit your manuscript to a SpringerOpen ${ }^{\circ}$ journal and benefit from:}

- Convenient online submission

Rigorous peer review

- Immediate publication on acceptance

Open access: articles freely available online

- High visibility within the field

- Retaining the copyright to your article 